\section{Colour tuning in human visual cortex measured with functional magnetic resonance imaging}

\author{
Stephen Engel* ${ }^{\star}$ Xuemei Zhang \& Brian Wandell \\ Stanford University, Department of Psychology, Building 420, Stanford, \\ California 94305, USA
}

The primate retina contains three classes of cones, the $L, M$ and $S$ cones, which respond preferentially to long-, middle- and shortwavelength visible light, respectively. Colour appearance results from neural processing of these cone signals within the retina and the brain. Perceptual experiments have identified three types of neural pathways that represent colour: a red-green pathway that signals differences between $L$ - and M-cone responses; a blueyellow pathway that signals differences between $S$-cone responses and a sum of L- and M-cone responses; and a luminance pathway that signals a sum of $\mathrm{L}$ - and $\mathrm{M}$-cone responses ${ }^{1-3}$. It might be expected that there are neurons in the primary visual cortex with response properties that resemble these three perceptual pathways, but attempts to find them have led to inconsistent results ${ }^{4-7}$. We have therefore used functional magnetic resonance imaging (fMRI) to examine responses in the human brain to a large number of colours. In visual cortical areas V1 and V2, the strongest response is to red-green stimuli, and much of this activity is from neurons receiving opposing inputs from $L$ and $M$ cones. A strong response is also seen with blue-yellow stimuli, and this response declines rapidly as the temporal frequency of the stimulus is increased. These responses resemble psychophysical measurements, suggesting that colour signals relevant for perception are encoded in a large population of neurons in areas $\mathrm{V} 1$ and V2.

Midget retinal ganglion cells and parvocellular neurons in the lateral geniculate nucleus (LGN) combine cone signals of opposite sign, as expected in chromatic pathways ${ }^{8-10}$. Several of the quantitative colour properties of neurons in the retina and LGN, however, do not match perceptual measurements. For example, perceptual spatial resolution is highest for luminance patterns, but the retinal mosaic with the highest spatial resolution, the midget ganglion cells, receive opposing input from the long-wavelength-sensitive (L) cones and the middle-wavelength-sensitive (M) cones, as expected in a chromatic pathway ${ }^{11,12}$.

Such discrepancies have focused attention on the cortex in an attempt to find neurons with properties that more closely resemble the three perceptual pathways ${ }^{7,10}$. However, single-unit colourtuning measurements in cortical area V1 have yielded conflicting results. Some measurements identify three populations of neurons, associated with particular cytochrome-oxidase blobs, that may correspond to the chromatic pathways ${ }^{5,13}$, but other results show broad distributions of colour properties, and yet others report that most V1 neurons respond best to luminance rather than chromatic patterns ${ }^{4,6,7}$.

To clarify the relationship between cortical activity and the perceptual colour pathways, we measured cortical colour tuning in areas $\mathrm{V} 1$ and $\mathrm{V} 2$ by using functional magnetic resonance imaging (fMRI). We used stimuli that reveal red-green and blue-yellow chromatic pathways in psychophysical experiments ${ }^{3,14,15}$, and measured colour tuning by using contrast-reversing 'checkerboard' patterns of many colours and contrasts. In each experiment,

* Present address: Department of Psychology, UCLA, Franz Hall, Los Angeles, California 90095, USA.
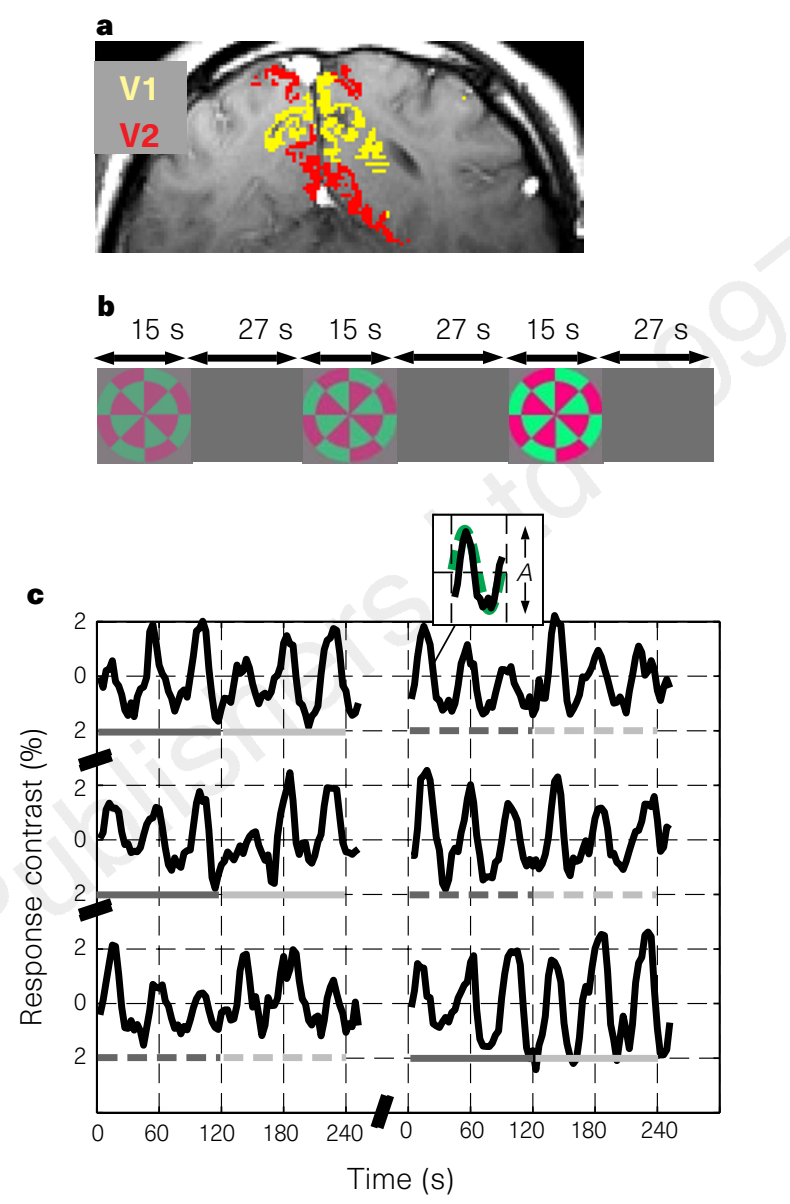

Figure 1 Experimental protocol. a, Cortical areas V1 (yellow) and V2 (red) (for observer B.W.). b. Stimulus time course (see Methods); only the central portion of the stimulus is shown (the actual stimulus contained many more checks). c, Typical time series of the fMRI signal (area V1, observer B.W., $4 \mathrm{~Hz}$, red-green plane). Six stimuli were presented during each $\mathrm{fMRI}$ scan. The lines beneath the time series are shaded to indicate stimuli with a common colour direction; solid and dotted lines denote increasing and decreasing contrast series, respectively. Inset shows response amplitudes $(A)$ to each stimulus, calculated by fitting harmonic functions to the time series.

observers viewed stimuli in six different colour directions at three levels of colour contrast (see Fig. 1). Each stimulus was presented for $15 \mathrm{~s}$ and was followed by a mean-field presentation for $27 \mathrm{~s}$; we call this composite presentation a stimulus period. The amplitude of the harmonic component of the fMRI signal with the stimulus period (42 s) served to measure the response of the neural population to each stimulus. This response increased monotonically with stimulus contrast. Each experiment was conducted with contrast-reversal temporal frequencies of 1,4 and $10 \mathrm{~Hz}$.

The first experiments measured colour tuning in a red-green stimulus plane in which checkerboards contained various levels of L- and M-cone contrast and zero S-cone contrast. Results for two observers are shown in Fig. 2. The solid iso-response contours show the L- and M-cone contrasts of stimuli that produce a criterion response amplitude. The dashed contours represent an $80 \%$ confidence interval surrounding the iso-response contour.

These data show that a large proportion of the response to chromatic stimuli arises from neurons that receive opposing Land M-cone signals. There are several ways to see the large contribution from such cells. First, the long straight edges of the iso-response contours fall along lines described by the equation $|L-M|=$ a constant, where $L$ and $M$ are the $\mathrm{L}$ - and $\mathrm{M}$-cone 


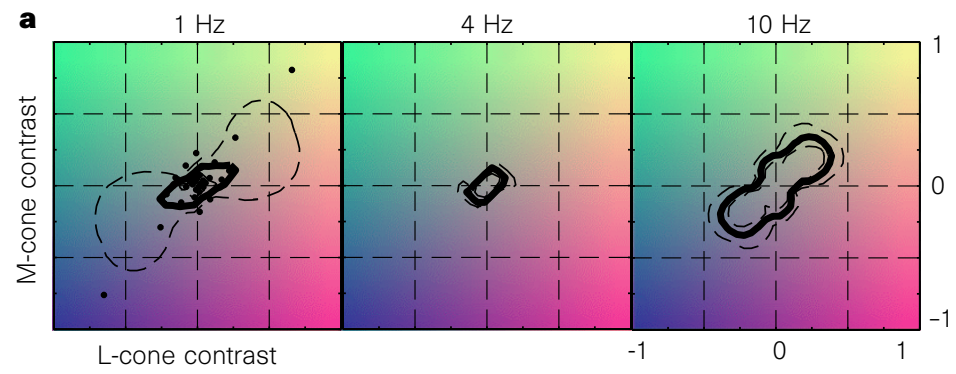

b

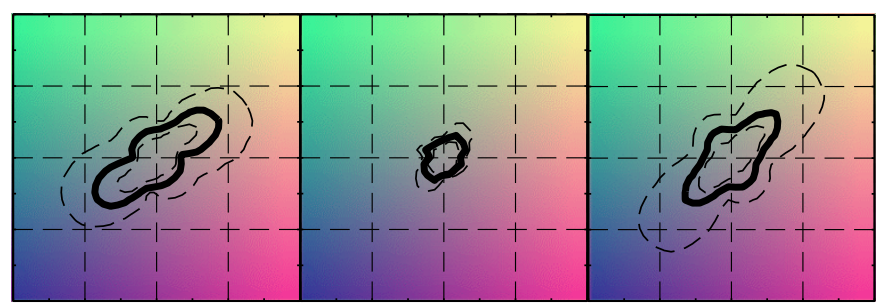

a

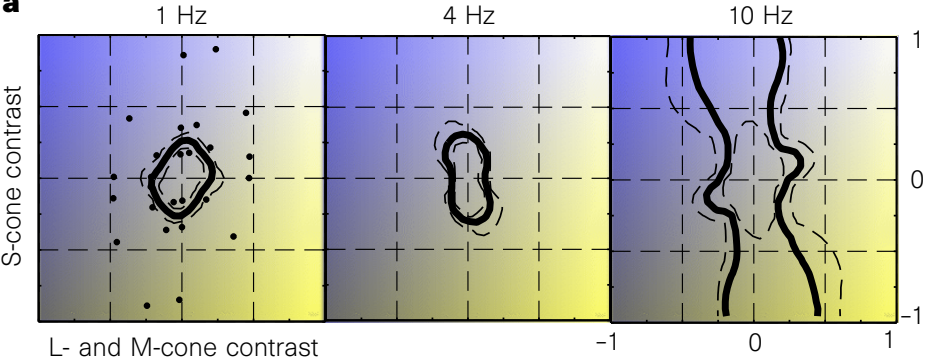

b

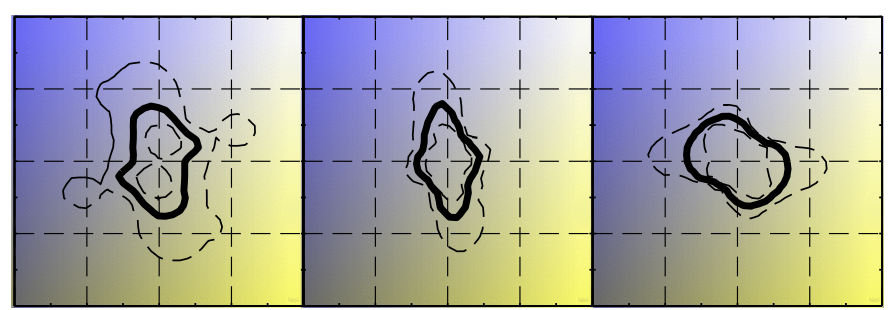

Figure 2 Colour tuning in a red-green plane measured in human cortical area V1. The solid contour shows the amount of cone contrast required to generate an fMRI response criterion level. The dotted contours represent $10 \%$ and $90 \%$ confidence intervals. The dots on the left in a show the L-and M-cone contrasts of the 18 different stimuli used in this experiment. The background colour shows the general appearance of the stimuli. The colour stimuli were presented at three temporal frequencies. Iso-response contours are shown for observer B.W. (a) and S.E. (b) measured using temporal frequencies of 1, 4 and $10 \mathrm{~Hz}$. For both observers, the response levels represented by the isoresponse contours are about twice as high at $4 \mathrm{~Hz}$ as at $1 \mathrm{~Hz}$ or $10 \mathrm{~Hz}$.

Figure 3 Colour tuning in a blue-yellow plane measured in human cortical area V2. The horizontal axis represents equal amounts of Land $\mathrm{M}$-cone contrast. The vertical axis represents S-cone contrast. Isoresponse contours (solid curves) for observer B.W. (a) and S.E. (b) at 1, 4 and $10 \mathrm{~Hz}$ temporal frequencies. For both observers, the response levels represented by the iso-response contours are about twice as high at $4 \mathrm{~Hz}$ as at $1 \mathrm{~Hz}$ or $10 \mathrm{~Hz}$. Other features are as Fig. 2. contrasts, respectively. Second, at all three temporal frequencies, more contrast is needed to obtain a given response level to $\mathrm{L}$ and $\mathrm{M}$ signals of common sign than of opposite sign; at $4 \mathrm{~Hz}$, for example, a cone contrast of roughly $(0.05,-0.05,0)$ yields the same response as a cone contrast of $(0.2,0.2,0)$. Hence, for this spatio-temporal pattern, cone contrast combined in opposite sign (a red-green chromatic stimulus) is four times more effective than cone signals combined in common sign (a luminance stimulus). Third, adding M-cone stimulation to a pure L-cone signal with the same sign reduces the $\mathrm{fMRI}$ response, creating a stimulus that falls inside the iso-response contour. Colour tuning measured in area V2 was virtually the same as colour tuning in area V1; the iso-response contours from V2 fell almost entirely within the confidence intervals obtained from area V1.

A second series of experiments measured colour tuning in a blueyellow plane, with stimulus axes specified in terms of S-cone contrast and combined equal amounts of L- and M-cone contrast. Iso-response contours in this plane from area V2 are shown in Fig. 3, and responses in V1 were very similar. At low temporal frequencies, we again obtained a robust fMRI signal in response to chromatic stimulation; responses in the blue-yellow $(1,1,-1)$ colour direction were equal to or greater than responses in the luminance direction $(1,1,0)$. The size of the blue-yellow signal is surprisingly large given that only $7 \%$ of the cones are of the S type, and that neurons in the LGN that principally respond to S-cone stimulation are relatively rare $^{16,17}$. As the temporal frequency of the stimulus increases, the relative size of the responses to stimuli in the blueyellow opponent direction and near the S-cone axis decreases compared to the responses to stimuli in the luminance directions.

We next compared the fMRI iso-response contours with psychophysical detection contours measured using the same checkerboard stimuli. The detection contours and individual threshold points for three temporal frequencies, two colour planes and one observer are shown in Fig. 4. Data from the other observer were in good agreement with these data, and both sets resemble previously published results $3,14,15,18,19$. The solid contours represent threshold stimulus contrasts, and the dashed contours represent an $80 \%$ confidence interval.

The perceptual data are similar to the cortical data in several respects. At 1 and $4 \mathrm{~Hz}$, in the red-green plane, sensitivity is highest to stimuli that cause opposing L- and M-cone signals. In the blueyellow plane, relatively high sensitivity to S-cone stimuli falls rapidly as temporal frequency increases.

The main difference between the cortical and behavioural 


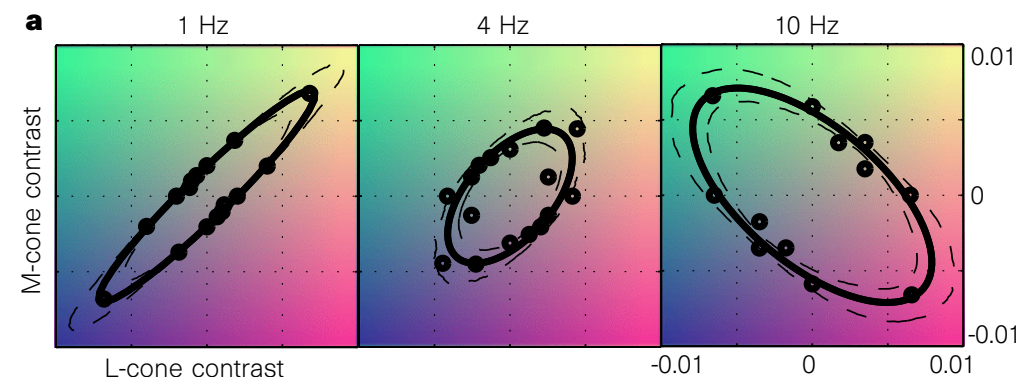

L-cone contrast

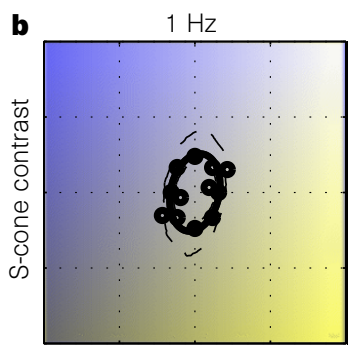

L- and M-cone contrast
$4 \mathrm{~Hz}$

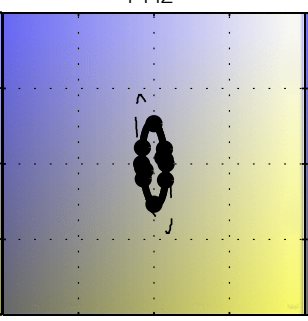

$10 \mathrm{~Hz}$
Figure 4 Psychophysical detection contours of the stimuli used in the fMRI experiments for observer B.W. The solid curve shows the detection threshold contour, and the open circles represent threshold contrast levels measured in various colour directions. The dotted curves represent a confidence interval, computed using a resampling procedure. $\mathbf{a}$, Data in a red-green plane; $\mathbf{b}$, data in a blue-yellow plane. Temporal frequencies at $1 \mathrm{~Hz}$ (left), $4 \mathrm{~Hz}$ (middle) and $10 \mathrm{~Hz}$ (right) are shown. Data for observer S.E. were similar. measurements is found for stimuli at $10 \mathrm{~Hz}$ in the red-green plane. The strongest fMRI response is to stimuli that produce opposing Land M-cone signals, but the behavioural sensitivity is best to stimuli in which the L- and M-cone signals covary. The fMRI data agree with preliminary electrophysiological data showing that some V1 neurons respond strongly to red-green stimuli at high temporal frequencies ${ }^{20,21}$. Perceptual sensitivity to these stimuli may be limited by processing beyond areas V1 and V2.

These fMRI measurements are consistent with measurements of visual evoked potential (VEP) that found larger responses to chromatic stimuli than to luminance stimuli of comparable contrast $^{22,23}$. The cortical sources of the VEP signal are unknown, however. The present results are also in agreement with fMRI measurements showing a larger V1 response to a red-green chromatic stimulus than to a luminance stimulus ${ }^{24}$. Our data extend these results by measuring complete colour-tuning curves, and comparing these results to behavioural data.

Our results indicate that cortical areas V1 and V2 are quite responsive to chromatic stimuli, and that the cortical responses resemble perceptual responses in important ways. These findings argue against the idea that perceptually relevant chromatic information is sparsely represented within V1 (refs 25,26$)$. The magnitude of the responses suggests that a relatively large population of neurons responds to the chromatic stimulation. The similarity between the cortical colour tuning and the behavioural colour sensitivity suggests that the cortical signals measured are relevant for colour perception. These kinds of colour-tuning measurements should allow us to learn how the spatiotemporal context, which influences colour perception in important ways, affects responses in the cortex.

\section{Methods}

Identification of a visual areas. Our methods for identifying visual areas are reported elsewhere in detail ${ }^{27-30}$; visual areas in the cortex were identified using measurements of retinotopic organization in the occipital lobe, viewed on flattened representations of cortex.

Scan planes. In all experiments, four or eight measurement planes of fMRI data were acquired using a blood oxygenation level-dependent pulse sequence (spiral $k$-space acquisition with echo time $(\mathrm{TE})=75$, repetition time $(\mathrm{TR})=300$, flip angle $(\mathrm{FA})=35$, pixel size $=1.5 \times 1.5 \times 5 \mathrm{~mm}$. One image per plane was acquired every $2.5 \mathrm{~s}$ or every $3 \mathrm{~s}$. Scan planes were oriented perpendicular to the calcarine sulcus and included the representation of the central $15 \mathrm{deg}$ of the visual field. T1-weighted anatomical images were taken in register with each functional scan plane. Subjects' heads were immobilized using a bite-bar; no motion artefacts were detected in our data.
Stimuli. Stimuli were circular, $20 \mathrm{deg}$, contrast-reversing, checkerboard patterns surrounded by a neutral mean field. The stimuli were imaged using an LCD video projector. We defined our stimuli in terms of cone contrast. Formally, let the values $L_{0}, M_{0}$ and $S_{0}$ represent the long-, medium- and shortwavelength cone absorptions from the mean field, and let $\Delta L, \Delta M$ and $\Delta S$, represent a perturbation of the stimulus checks from the mean value. The stimulus is represented by the three-dimensional cone contrast vector, $s=\left(\Delta L / L_{0}, \Delta M / M_{0}, \Delta S / S_{0}\right)$. Alternate checks were equal positive and negative perturbations. We define the colour contrast of the stimulus to be the vector length, $\|s\|$. The colour direction of the stimulus is unit length vector $s /\|s\|$. Presentation of stimuli. Stimuli were presented in triplets with a common colour direction; the colour contrast between consecutive members of a triplet increased by a factor of two. Stimuli in each colour direction were presented twice, once in increasing order by contrast, and once in decreasing order. Six stimuli were presented during each fMRI scan.

fMRI contours. Iso-response contours were estimated using the average fMRI responses from all pixels in a cortical area. A criterion response was chosen that was approximately half of the maximum response measured in the experiment. Contours represent the interpolated set of stimuli that produce the criterion level of response. Confidence intervals were generated using a resampling procedure. We reanalysed our data 500 times, using random draws with replacement from our original data. Stimuli inside the outer contour produced responses greater than the criterion in $90 \%$ of the resamplings; stimuli inside the inner contour produced such responses in $10 \%$ of the resamplings.

Detection contours. Perceptual thresholds were measured using a display controlled by a 30-bit frame buffer. The probability of detecting each of many stimuli, which differed in colour direction and contrast, was measured using a two-interval, forced-choice, multiple staircase design. Thresholds for individual colour directions and the full elliptical detection contours were estimated using a maximum-likelihood procedure. The detection contours and confidence intervals represent estimates based on more than 1,000 trials. The inner and outer confidence contours represent $10 \%$ and $90 \%$ levels, based on the same resampling procedure used to analyse the fMRI data.

Received 17 January; accepted 30 April 1997.

1. Hurvich, L. \& Jameson, D. An opponent-process theory of color vision. Psychol. Rev. 64, 384-404 (1957).

2. Boynton, R. et al. Interactions among chromatic mechanisms as inferred from positive and negative increment thresholds. Vis. Res. 4, 87-117 (1964).

3. Thornton, J. \& Pugh, E. Red/green color opponency at detection threshold. Science 219, 191-183 (1983).

4. Thorell, L. et al. Spatial mapping of monkey V1 cells with pure color and luminance stimuli. Vis. Res. 24, 751-769 (1984).

5. Livingstone, M. \& Hubel, D. Anatomy and physiology of a colour system in the primate visual cortex. J. Neurosci. 4, 2830-2835 (1984).

6. Vautin, R. \& Dow, B. Color cell groups in foveal striate cortex of the behaving macaque. $J$. Neurophysiol. 54, 273-292 (1985).

7. Lennie, P. et al. Chromatic mechanisms in striate cortex of macaque. J. Neurosci. 10, 649-669 (1990). 
8. DeValois, R. L. Analysis and coding of color vision in the primate visual system. Cold Spring Harb. Symp. Quant. Biol. 30, 567-579 (1965).

9. Wiesel, T. \& Hubel, D. S. Spatial and chromatic interactions in the lateral geniculate body of the rhesus monkey. J. Neurophysiol. 29, 1115-1156 (1966).

10. Derrington, A. et al. Chromatic mechanisms in lateral geniculate nucleus of macaque. J. Physiol. (Lond.) 357, 241-265 (1984).

11. Mullen, K. The contrast sensitivity of human colour vision to red-green and blue-yellow chromatic gratings. J. Physiol. (Lond.) 359, 381-400 (1985).

12. Poirson, A. \& Wandell, B. Appearance of colored patterns: Pattern-color separability. J. Opt. Soc. Am $12,2458-2471$ (1993).

13. Ts'o, C. \& Gilbert, C. The organization of chromatic and spatial interactions in the primate striate cortex. J. Neurosci. 8, 1712-1727 (1988).

14. Wandell, B. Color measurement and discrimination. J. Opt. Soc. Am. A 2, 62-71 (1985).

15. Cole, G. et al. Detection of mechanisms in L-, M-, and S-cone contrast space. J. Opt. Soc. Am. A 10, 38 51 (1993).

16. Curcio, C. A. et al. Distribution and morphology of human cone photoreceptors stained with antiblue opsin. J. Comp. Neurol. 312, 610-624 (1991).

17. Dacey, D. M. \& Lee, B. B. The 'blue-on' opponent pathway in primate retina originates from a distinct bistratified ganglion cell type. Nature 367, 731-735 (1994).

18. Kelly, D. Theory of flicker and transient responses: I. Uniform fields. J. Opt. Soc. Am. 61, 537-546 (1971)

19. Stockman, A. The temporal properties of the human short-wave photoreceptors and their associated pathways. Vis. Res. 31, 189-208 (1991).

20. Cottaris, N. P. et al. Spatio-temporal luminance and chromatic receptive field profiles of macaque striate cortex simple cells. Soc. Neurosci. Abstr. 22, 951 (1996).

21. Gur, M. \& Snodderly, D. M. A dissociation between brain activity and perception: Chromatically opponent cortical neurons signal chromatic flicker that is not perceived. Vis. Res. 37, 377-382 (1997)

22. Regan, D. Evoked potentials specific to spatial patterns of luminance and colour. Vis. Res. 13, 23812402 (1973)

23. Rabin, J. et al. Visual evoked potentials in three-dimensional color space: Correlates of spatiochromatic processing. Vis. Res. 34, 2657-2671 (1994).

24. Kleinschmidt, A. et al. Functional mapping of color processing by magnetic resonance imaging of responses to selective P- and M-pathway stimulation. Exp. Brain Res. 110, 279-288 (1996).

25. Rodieck, R. W. in From Pigments to Perception (eds Valberg, A. \& Lee, B. B.) 83-93 (Plenum, New York, 1991).

26. Calkins, D. J. \& Sterling, P. Absence of spectrally specific lateral inputs to midget ganglion cells in primate retina. Nature 381, 613-615 (1996).

27. Engel, S. A. et al. fMRI of human visual cortex. Nature 369, 525 (1994).

28. Sereno, M. et al. Borders of multiple visual areas in humans revealed by functional magnetic resonance imaging. Science 268, 889-893 (1995).

29. DeYoe, E. et al. Mapping striate and extrastriate visual areas in human cerebral cortex. Proc. Natl Acad. Sci. USA 93, 2382-2386 (1996).

30. Engel, S. A. et al. Retinotropic organization in human visual cortex and the spatial precision of functional MRI. Cereb. Cort. 7, 181-192 (1997).

Acknowledgements. We thank G. Glover for technical help, and H. Baseler, G. Boynton, E. J. Chichilnisky E. Markman, W. Newsome, A. Poirson and J. Wine for comments on the manuscript. This work was funded by the National Eye Institute and the McDonnel-Pew Foundation.

\section{Hippocampal GABA $A_{A}$ channel conductance increased by diazepam}

\section{Eghbali, J. P. Curmi, B. Birnir \& P. W. Gage}

John Curtin School of Medical Research, PO Box 334, Australian National University, Canberra, ACT 2601, Australia

Benzodiazepines, which are widely used clinically for relief of anxiety and for sedation ${ }^{1}$, are thought to enhance synaptic inhibition in the central nervous system by increasing the open probability of chloride channels activated by the inhibitory neurotransmitter $\gamma$-aminobutyric acid (GABA) ${ }^{2,3}$. Here we show that the benzodiazepine diazepam can also increase the conductance of $\mathrm{GABA}_{\mathrm{A}}$ channels activated by low concentrations of GABA $(0.5$ or $5 \mu \mathrm{M})$ in rat cultured hippocampal neurons. Before exposure to diazepam, chloride channels activated by GABA had conductances of 8 to $53 \mathrm{pS}$. Diazepam caused a concentration-dependent and reversible increase in the conductance of these channels towards a maximum conductance of 70$80 \mathrm{pS}$ and the effect was as great as 7-fold in channels of lowest initial conductance. Increasing the conductance of $\mathrm{GABA}_{\mathrm{A}}$ channels tonically activated by low ambient concentrations of GABA in the extracellular environment ${ }^{4}$ may be an important way in which these drugs depress excitation in the central nervous system. That any drug has such a large effect on single channel conductance has not been reported previously and has implications for models of channel structure and conductance.
Cultures of hippocampal neurons from neonatal rats were prepared as described previously ${ }^{5}$ and single-channel currents activated by 0.5 or $5 \mu \mathrm{M}$ GABA were recorded in cell-attached, inside-out or outside-out patches. Currents recorded had properties typical of GABA-activated chloride channels in these cells ${ }^{6-8}$ : they reversed at a pipette potential close to $0 \mathrm{mV}$ even when $\mathrm{Na}^{+}$was replaced by $\mathrm{K}^{+}$in the pipette solution, showed outward rectification, had conductances that varied over a wide range and were blocked by injection of $100 \mu \mathrm{M}$ bicuculline into the pipette tip. In 27 patches in which single-channel currents were recorded before and after exposure to diazepam, single-channel conductance (current amplitude divided by the difference between the pipette potential $(-40$ to $-80 \mathrm{mV})$ and the reversal potential) before exposure of a patch to diazepam ranged from 8 to $53 \mathrm{pS}$ (Table 1). This is a wide variation but within the range of conductances of $\mathrm{GABA}_{\mathrm{A}}$ channels ${ }^{9,10}$.

The binding site for benzodiazepines is thought to involve residues in the extracellular domain of $\alpha$ and perhaps $\gamma$-subunits ${ }^{11}$. When diazepam was applied to the extracellular surface of cellattached patches by injecting the drug into the pipette tip ${ }^{6}$, an increase in the amplitude of single-channel currents activated by GABA was seen. In Fig. 1, single channel currents activated by $5 \mu \mathrm{M}$ GABA (Fig. 1a) had an average amplitude of $0.9 \mathrm{pA}$ (pipette potential of $-80 \mathrm{mV}$ ), as can be seen in the all-points histogram in Fig. 1c. Following injection of a bolus of control solution containing $5 \mu \mathrm{M}$ GABA plus $10 \mu \mathrm{M}$ diazepam into the pipette tip, the single-channel currents (Fig. 1b) showed more than a fourfold increase in amplitude, giving a single open peak in the all-points
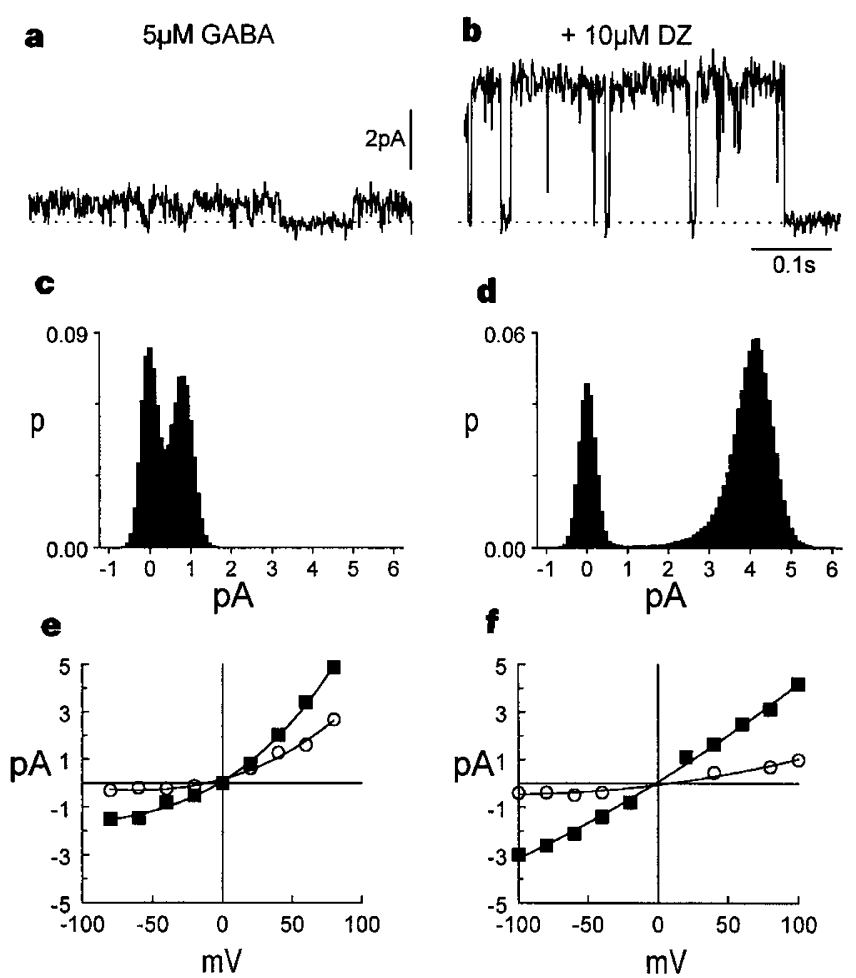

Figure 1 The increase in single-channel current amplitude caused by $10 \mu \mathrm{M}$ diazepam in a cell-attached patch (pipette potential of -80 mV). a, Single-channel currents activated by $5 \mu \mathrm{M}$ GABA. b. Single-channel currents recorded from the same patch after injection of a solution containing $5 \mu \mathrm{M}$ GABA and $10 \mu \mathrm{M}$ diazepam into the pipette tip. c. All-points histogram of a 16-s current recording, including the segment in a. d. All-points histogram of a 16-s current recording including the segment in b.e, Current-voltage relation for single-channel currents activated by $0.5 \mu \mathrm{M}$ GABA in a cell-attached patch before (circles) and after (squares) injection of $1 \mu \mathrm{M}$ diazepam. f, Current-voltage relation for singlechannel currents activated by $0.5 \mu \mathrm{M}$ GABA in an inside-out patch before (circles) and after (squares) exposure to $1 \mu \mathrm{M}$ diazepam. 